\title{
Association between prenatal exposure to perfluorinated compounds and symptoms of infections at age 1-4years among 359 children in the Odense Child Cohort
}

\section{Citation}

Dalsager, Louise, Nikolas Christensen, Steffen Husby, Henriette Kyhl, Flemming Nielsen, Arne Høst, Philippe Grandjean, and Tina Kold Jensen. 2016. "Association Between Prenatal Exposure to Perfluorinated Compounds and Symptoms of Infections at Age 1-4years Among 359 Children in the Odense Child Cohort." Environment International 96 (November): 58-64. doi:10.1016/ j.envint.2016.08.026.

\section{Published Version}

doi:10.1016/j.envint.2016.08.026

\section{Permanent link}

http://nrs.harvard.edu/urn-3:HUL.InstRepos:37221746

\section{Terms of Use}

This article was downloaded from Harvard University's DASH repository, and is made available under the terms and conditions applicable to Open Access Policy Articles, as set forth at http:// nrs.harvard.edu/urn-3:HUL.InstRepos:dash.current.terms-of-use\#OAP

\section{Share Your Story}

The Harvard community has made this article openly available.

Please share how this access benefits you. Submit a story.

\section{Accessibility}




\section{Association between prenatal exposure to perfluorinated compounds and symptoms of infections at age 1-4 years among 359 children in the Odense Child Cohort}

Louise Dalsager ${ }^{1,2}$, Nikolas Christensen ${ }^{3,4}$, Steffen Husby ${ }^{3}$, Henriette Kyhl ${ }^{3,5}$, Flemming Nielsen $^{1}$, Arne Høst ${ }^{3}$, Philippe Grandjean ${ }^{1,6}$, Tina Kold Jensen ${ }^{1,2}$

${ }^{1}$ Department of Environmental Medicine, Institute of Public Health, University of Southern Denmark, Odense, J.B. Winsløwsvej 17A, 5000 Odense, Denmark ${ }^{2}$ Rigshospitalet, Copenhagen University Hospital, Department of Growth and Reproduction, Blegdamsvej 9, 2100 Copenhagen, Denmark

${ }^{3}$ Hans Christian Andersen Children's Hospital, Odense University Hospital, Sdr. Boulevard 29, 5000 Odense C, Denmark.

${ }^{4}$ Department of Clinical Research, Faculty of Health Sciences, University of Southern Denmark, Odense, Denmark.

${ }^{5}$ Odense Patient data Exploratory Network (OPEN), Odense, Denmark

${ }^{6}$ Department of Environmental Health, Harvard T.H. Chan School of Public Health, Boston, MA, USA

\section{Corresponding author:}

Louise Dalsager, Department of Environmental Medicine, Institute of Public Health, University of Southern Denmark, Winsløwsparken 17, 5000 Odense, Denmark. Telephone: +4528195273, E-mail: 1dalsager@health.sdu.dk.

Short running title: Prenatal exposure to PFAS and infectious symptoms

\section{Acknowledgements:}

The technicians at Hans Christian Andersen's Children's Hospital are acknowledged for their careful examination of the children. This work was supported by the Danish Center for Hormone Disrupting Chemicals, the Danish Foundation for Scientific Innovation and Technology (09-067180), The Danish Research Council (4004-00352B_FSS), Ronald 
McDonald Children Foundation, K. A. Rohde's and wife's Foundation, Odense University Hospital and Region of Southern Denmark, Municipality of Odense, Danielsen Foundation, The Danish Council for Strategic Research, Program Commission on Health, Food and Welfare (2101-08-0058), Odense University Hospital Research Foundation and Odense Patient data Exploratory Network (OPEN).

Competing financial interests: None 


\begin{abstract}
Introduction: Perfluorinated alkylated substances (PFAS) are persistent industrial chemicals that have resulted in global environmental exposures. Previous epidemiological studies have reported possible effects on the immune system after developmental PFAS exposure, but the possible impact on childhood infectious disease is unclear.
\end{abstract}

Objectives: To investigate the association between prenatal exposure to PFAS and symptoms of infections at age 1-4 years.

Methods: The Odense Child Cohort is an on-going prospective study on children's health, where serum concentrations of perfluorooctane sulfonic acid (PFOS), perfluorooctanoic acid (PFOA), perfluorohexane sulfonic acid (PFHxS), perfluorodecanoic acid (PFDA) and perfluorononanoic acid (PFNA) were measured in 649 pregnant women before gestational week 16. Of these women, 359 reported on symptoms of infection in their child every two weeks for a one-year period. The association between prenatal exposure to PFAS and the symptoms was estimated using a logistic regression model and a negative binomial regression model. For the latter, the outcome was reported as an incidence rate-ratio (IRR), and all models were adjusted for maternal age, educational level, parity and child age.

Results: On average, and accounting for incomplete reporting, the children experienced symptoms of infection $23 \%$ of the time during one year. PFOS exposure in the high tertile compared to the low tertile was associated with a statistically significant increased proportion of days with fever (IRR: 1.65 (95\% CI: 1.24, 2.18), p-trend<0.001) and an increased odds of experiencing days with fever above the median (OR: 2.35 (95\%CI: 1.31, 4.11). The latter tendency was also apparent for PFOA (OR: 1.97 (95\%CI: 1.07, 3.62). Further, higher concentrations of PFOS and PFOA tended to increase the number of episodes of cooccurrence of fever and coughing and fever and nasal discharge during the one-year study period. 
Conclusion: We found a positive association between prenatal exposure to PFOS and PFOA and the prevalence of fever, which may be a sensitive marker of infection. This finding is in agreement with an immunotoxic effect of prenatal exposure to PFAS. The wider implications for childhood infectious disease deserve attention.

Keywords: perfluorinated compounds, childhood infections, immunotoxicity, prenatal exposure, cohort study 


\section{Introduction}

Perfluorinated compounds (PFASs) are highly persistent industrial chemicals that have resulted in global environmental dissemination (Lau et al. 2007). The most common types are PFOS (perfluorooctane sulfonic acid), and PFOA (perfluorooctanoic acid), which are ubiquitously present in human serum, where they show an estimated elimination half-life of four years for PFOA and five years for PFOS (Calafat et al. 2007; Kato et al. 2011; Olsen et al. 2007). They cross the placenta (Needham et al. 2011) and are released through human milk (Mogensen et al. 2015), thereby causing early-life exposure that may be worthy of concern. Due to changes in production, serum concentrations of some PFASs have decreased, although others have increased, including perfluorohexane sulfonic acid (PFHxS,) perfluorononanoic acid (PFNA), and perfluorodecanoic acid (PFDA) (Glynn et al. 2012). Among potential toxic effects, developmental PFAS exposures have been linked to lower efficiency of routine childhood immunizations (Grandjean et al. 2012; Granum et al. 2013). Increased prevalence of self-reported common cold and gastroenteritis was found during the first three years of life in children with elevated prenatal exposure to PFOA, PFNA and PFHxS in utero (Granum et al. 2013). However, among 1400 members of the Danish Birth Cohort, hospitalization for infectious diseases such as appendicitis or pneumonia was not associated with maternal PFOA and PFOS concentrations in serum during the first eleven years of life (Fei et al. 2010). On the other hand, a recent report showed that the PFAS analyses in this study might have been imprecise (Bach et al. 2015), thus limiting the validity of this finding. A key concern in these studies is how infection is defined and reported. In children, fever is an almost universal sign of infection, and other causes of elevated body temperature are rare in temperate countries (Sullivan and Farrar 2011). 
The aim of this study was therefore to investigate the association between pregnancy serum concentrations of PFOS, PFOA, PFDA, PFNA and PFHxS and fever and other symptoms of infections during a one-year period among children aged 1-4 years.

\section{Methods}

\subsection{Study participants and data collection}

The participants were derived from the Odense Child Cohort. Briefly, all women living in the municipality of Odense who were pregnant between January $1^{\text {st }} 2010$ and December $31^{\text {st }}$ 2012 were invited to participate (a total of 6,707). They were recruited either at a voluntary meeting about ultrasound examinations, at their first antenatal visit, or at the ultrasound examination at Odense University Hospital between gestational age (GA) 10-16 weeks (Kyhl et al. 2015). Of the eligible women, 4,017 were informed and 2,874 (43\%) agreed to participate. Today 2,446 singleton children are still being followed. At the time of inclusion, a blood sample was drawn and the participants filled out a questionnaire on general health, lifestyle and social factors. Questionnaires focusing on the child's health and well-being were completed at age three months, 18 months and 3 years, and together with the birth records, these were the sources of data on co-variables. Information on maternal age and educational level was obtained from the questionnaire completed at the time of inclusion, while information on parity, smoking and child sex was derived from the birth record. Educational level was missing in the questionnaires for 119 women and the information was then retrieved from the obstetric records. Duration of breastfeeding and day-care attendance were reported in the questionnaire at age 18 months.

\subsection{PFAS measurements}

From the serum samples collected, a subset of 649 samples was analyzed for PFAS. Of these, 
200 were selected randomly while the remaining 449 were selected based on the availability of information from questionnaires, birth records, and a clinical examination of the child at three months of age. The blood sample preparation and storage were uniform, as appropriate for PFAS analysis (Kato et al. 2013). After centrifugation, serum was pipetted into polypropylene cryo tubes and immediately frozen to $-80^{\circ} \mathrm{C}$ and stored up to three years until analysis. As is standard in this field, we specifically avoided using sampling or storage tubes containing fluoropolymers (such as Teflon-coated materials) that could be a source of PFAS contamination (Berger et al. 2011). Although sample contamination or adsorption is always a possibility, our repeated analyses of serum samples over time as well as analyses of duplicate samples have shown excellent precision and have never revealed any indication of variance attributable to sampling and storage (Jensen et al. 2015). The serum-PFAS concentrations were analyzed using on-line solid phase extraction followed by liquid chromatography and triple quadropole mass spectrometry (LC-MS/MS) at Environmental Medicine, University of Southern Denmark. The quantified PFASs include perfluorooctanesulfonic acid (PFOS), perfluorooctanoic acid (PFOA), perfluorohexane sulfonic acid (PFHxS), perfluorononanoic acid (PFNA), and perfluorodecanoic acid (PFDA). For all compounds, the Limit of Quantification (LOQ) was $0.03 \mathrm{ng} / \mathrm{mL}$. Results from the first 200 samples as well as more detailed information of PFAS analyses have been previously published (Vorkamp et al. 2014).

\subsection{Symptoms of infection in children}

In June 2014, 1,647 of the 2,547 families, who were enrolled and active in the Odense Child Cohort at that time, were invited to participate in a study of childhood infections in which symptoms of infection had to be reported by text messages every second week (26 times) during one year. At the time of invitation the children were between 1.0 and 3.3 years old. It 
was not possible to invite all 2,547 due to a clerical error in the informed consent materials. Of the families invited, a total of $1,540(93 \%)$ accepted to participate. Symptoms of infection were assessed using mobile-phone questionnaires (SMS-Track Aps, Esbjerg, Denmark). The participants received a text message every second Sunday and were asked to evaluate the occurrence of 11 symptoms during the previous two weeks. The parents reported every symptom with a value between zero and 14 reflecting the number of days the symptom had been present within the two-week period. Data on the following symptoms were collected: days without symptoms, fever, stuffed or runny nose, cough, wheezy or whistling breathing, eye inflammation, ear pain, discharge from ear, feeling unwell, diarrhea, blood in stool, and vomiting. All parents were provided with written information on when and how to report the symptoms. In the present study, we focused on fever, cough, nasal discharge, diarrhea and vomiting. Fever was considered the most relevant outcome for assessing infection, but the other symptoms were included as likely symptoms of common cold and gastroenteritis, which have previously been found to be associated with three of the five PFASs investigated here (Granum et al. 2013). Participants were instructed that a rectal temperature above $38.5^{\circ} \mathrm{C}$ was required to be certain about the presence of fever.

The study was performed in accordance with the second Helsinki Declaration and approved by the regional Ethical Review Committee (Project ID S-20090130). All participants received written and oral information and gave their written consent.

\subsection{Statistical analysis}

Due to a non-normal distribution of the serum-PFAS concentrations, these were logtransformed and reported as medians. The PFAS concentrations were examined in regard to relevant characteristics of the participants, and T-tests and F-tests were conducted. 
Characteristics of participants included in the analysis were compared to those who dropped out using a $\mathrm{T}$-test for continuous variables and $\mathrm{Chi}^{2}$ test for categorical variables.

The reported number of days with each symptom was summarized for all 26 periods to create a mean for the whole year. Since not all participants answered the 26 text messages and therefore did not report the presence of symptoms for all days during the whole year, the time at risk differed. This was taken into account by calculating the proportion of days with a given symptom out of the total number of days for which information on symptoms were provided. Further, to quantify the extent of the missing replies to the text messages, the mean reply rate for the 26 periods was calculated.

The associations between the PFAS exposures and the symptoms of infection were assessed using regression models, and the outcomes were analyzed both as dichotomous and ordinal data. First, the number of days with a symptom was transformed into a binary variable reflecting whether or not a child's proportion of days with a symptom during the last year was above or below the median proportion of days with the symptom (Model 1). These data were analyzed using logistic regression to obtain an odds ratio, which expresses the relative difference between the odds of two people who only differ in one parameter. Second, the number of days with a symptom was analyzed as ordinal data using a negative binomial regression (Model 2), since the variability in the data was greater than expected from a Poisson distribution. Additionally, the number of co-occurrences of two or more symptoms (fever and coughing or nasal discharge) within the same 14-days period during the one-year period was analyzed by the same approach (Model 3). Time at risk differed between participants and was therefore included in the model. The estimates produced by this approach were incidence rate-ratios, which in this study reflect the relative difference between the expected proportions of days with symptoms when comparing two groups 
(Model 2) and the relative difference between the expected proportion of 14-day periods with co-occurrence of more symptoms when comparing two groups (Model 3).

For both the logistic and the negative binomial regression analyses, the serum-PFASs were divided into tertiles, and for the negative binomial regression, a test for a linear trend across the exposure groups was conducted. Potential confounders of the association were found through review of the literature, and assessed using a directed acyclic graph (DAG) (Hernán and Robins 2015). Factors a priori found to be predictive of both exposure and outcome were considered possible confounders and included in the multivariate models. These included maternal age (categorical: $<25,25-29,>29$ years), educational level (categorical: high school or less, high school + 1-4 years, high school + more than 4 years), and parity (dichotomous: $0,>0)$.

Further, all models were adjusted for child age at the beginning of the study, since age was expected to be associated with childhood infections and with postnatal PFAS exposure. As PFAS concentrations were highly correlated, mutual adjustment was not attempted.

Since not all participants responded to the complete set of 26 text-messages, the analyses were repeated for those who responded to at least 25 of 26 text-messages ( $N=148)$. Finally, analyses were repeated after additional adjustment for maternal smoking (yes/no), child sex, day-care attendance (yes/no) and exclusive breastfeeding (continuous, number of weeks), even though PFAS exposure recently was found to be associated with decreased duration of breastfeeding and thus may be an intermediary factor (Romano et al. 2016; Timmermann et al. 2016).

The assumptions underlying both the negative binomial regression model and the logistic regression model were examined and accepted. As general significance level, we used $p=$ 0.05; given the multiple comparisons, a Bonferroni adjusted significance level $(\alpha=0.002)$ was also considered. 


\section{Results}

Of the 1,540 families who agreed to participate in this substudy on infections, 439 had PFAS concentrations measured during early pregnancy and thus composed the study population. Eighty participants were excluded during the study period; thirty-six due to no reply within the first 1.5 months and 44 due to lack of response to at least four consecutive text messages when the study had been running for 7 months. This left 359 participants. The 80 excluded mothers did not differ from the participating mothers according to serum-PFAS concentrations, educational level and parity but were younger (data not shown). During each 14-day period an average of $86 \%$ (range: $79.9-92.8 \%$ ) of the 359 participants replied to the text message, whereas $23 \%$ answered all text messages during the 26 periods.

Baseline characteristics of the participants including maternal serum concentrations of PFAS are shown in Table 1. The mean age of the participating mothers was $30.5( \pm 4.4 \mathrm{SD})$ years, $59 \%$ were nulliparous and $20 \%$ were in the highest educational group. The mean prepregnancy BMI was $24.6( \pm 4.4) \mathrm{kg} / \mathrm{m}^{2}$ and $3 \%$ smoked during pregnancy. Of the children, $54 \%$ were boys, $3.6 \%$ were preterm and the mean birth weight was $3,557( \pm 540)$ grams. On average the duration of exclusive breastfeeding was $19.2( \pm 8.1)$ weeks and at age 18 months the majority of children were attending day-care (82\%).

The concentrations of PFOS, PFOA, PFHxS and PFNA were significantly higher in nulliparous compared to multiparous women. Further, the PFAS concentrations tended to decrease with both increasing maternal age and increasing educational level, which was statistically significant for both PFOS and PFOA. An increase in pre-pregnancy BMI was associated with lower PFDA concentrations and the same tendency was seen for the remaining PFAS. Finally, higher maternal serum concentrations of the compounds were seen when the child was older (this was opposite for PFHxS) and when the child was a boy. 


\begin{tabular}{|c|c|c|c|c|c|c|}
\hline & $\mathbf{N}^{\mathrm{a}}(\%)$ & PFOS & PFOA & PFHxS & PFNA & PFDA \\
\hline Median & $359(100)$ & 8.07 & 1.68 & 0.32 & 0.70 & 0.27 \\
\hline Range (min.-max) & & $2.36-25.10$ & $0.32-10.12$ & $0.02-1.03$ & $0.21-3.64$ & $0.10-1.67$ \\
\hline \multicolumn{7}{|c|}{ Maternal characteristics during pregnancy } \\
\hline \multicolumn{7}{|c|}{ Age (years) } \\
\hline$<25$ & $31(8.6)$ & $10.06^{*}$ & $1.90^{*}$ & 0.32 & 0.82 & 0.29 \\
\hline $25-29$ & $123(34.3)$ & 8.21 & 1.87 & 0.31 & 0.72 & 0.28 \\
\hline$>29$ & $200(55.7)$ & 7.52 & 1.50 & 0.32 & 0.64 & 0.26 \\
\hline Missing & $5(1.4)$ & & & & & \\
\hline \multicolumn{7}{|l|}{ Pre-pregnancy BMI $\left(\mathrm{kg} / \mathrm{m}^{2}\right)$} \\
\hline$<20$ & $31(8.6)$ & 9.66 & 1.87 & 0.34 & 0.73 & $0.31 *$ \\
\hline $20-24.9$ & $187(52.1)$ & 8.13 & 1.68 & 0.32 & 0.72 & 0.28 \\
\hline$>25$ & $136(37.9)$ & 7.73 & 1.64 & 0.31 & 0.64 & 0.26 \\
\hline Missing & $5(1.4)$ & & & & & \\
\hline \multicolumn{7}{|l|}{ Smoking } \\
\hline Yes & $11(3.1)$ & 7.95 & 1.92 & 0.30 & 0.57 & 0.28 \\
\hline No & $343(95.5)$ & 8.12 & 1.68 & 0.32 & 0.70 & 0.24 \\
\hline Missing & $5(1.4)$ & & & & & \\
\hline \multicolumn{7}{|l|}{ Educational level } \\
\hline High school or less & $99(27.6)$ & $8.51^{*}$ & $1.87^{*}$ & 0.32 & 0.73 & 0.29 \\
\hline High school + 1-4 years & $180(50.1)$ & 8.32 & 1.61 & 0.30 & 0.68 & 0.27 \\
\hline High school $+>4$ years & $73(20.3)$ & 7.26 & 1.50 & 0.32 & 0.71 & 0.27 \\
\hline Missing & $7(1.9)$ & & & & & \\
\hline \multicolumn{7}{|l|}{ Parity } \\
\hline Nulliparous & $211(58.8)$ & $8.85^{*}$ & $2.01^{*}$ & $0.36^{*}$ & $0.75^{*}$ & 0.28 \\
\hline Multiparous & $143(39.8)$ & 7.08 & 1.24 & 0.24 & 0.59 & 0.25 \\
\hline Missing & $5(1.4)$ & & & & & \\
\hline \multicolumn{7}{|l|}{ Child characteristics } \\
\hline \multicolumn{7}{|l|}{ Sex } \\
\hline Boy & 194(54) & 8.36 & $1.75^{*}$ & $0.33^{*}$ & 0.71 & 0.27 \\
\hline Girl & $165(46)$ & 7.72 & 1.57 & 0.29 & 0.66 & 0.27 \\
\hline Missing & 0 & & & & & \\
\hline \multicolumn{7}{|l|}{ Age at inclusion (years) } \\
\hline 1 & $59(16.4)$ & 7.52 & 1.68 & $0.30^{*}$ & $0.57^{*}$ & 0.26 \\
\hline 2 & $212(50.1)$ & 8.04 & 1.67 & 0.33 & 0.76 & 0.28 \\
\hline 3 & $82(22.8)$ & 8.43 & 1.74 & 0.22 & 0.62 & 0.27 \\
\hline Missing & $6(1.7)$ & & & & & \\
\hline \multicolumn{7}{|l|}{$\begin{array}{l}\text { Attending day-care at age } 18 \\
\text { months }\end{array}$} \\
\hline Yes & 293(81.6) & 8.21 & 1.71 & 0.32 & 0.71 & 0.27 \\
\hline No & $5(1.4)$ & 8.55 & 1.68 & 0.37 & 0.71 & 0.37 \\
\hline Missing & $61(17.0)$ & & & & & \\
\hline \multicolumn{7}{|l|}{$\begin{array}{l}\text { Exclusive breastfeeding } \\
\text { (weeks) }\end{array}$} \\
\hline$<4$ & $112(31.2)$ & 7.82 & 1.55 & 0.32 & 0.64 & 0.25 \\
\hline $4-19$ & $103(28.7)$ & 7.86 & 1.70 & 0.30 & 0.70 & 0.27 \\
\hline$>19$ & $144(40.1)$ & 8.34 & 1.70 & 0.32 & 0.71 & 0.28 \\
\hline Missing & 0 & & & & & \\
\hline \multicolumn{7}{|l|}{ Birth weight (grams) } \\
\hline$<2500$ & $6(1.7)$ & 7.62 & 1.82 & 0.36 & 0.57 & 0.25 \\
\hline $2500-4499$ & $334(93.0)$ & 8.17 & 1.70 & 0.32 & 0.70 & 0.28 \\
\hline$>4499$ & 14(3.9) & 7.38 & 1.47 & 0.27 & 0.64 & 0.28 \\
\hline
\end{tabular}




\begin{tabular}{ccccccc} 
Missing & $5(1.4)$ & & & & & \\
Preterm $(<37$ weeks $)$ & & 7.52 & 1.87 & 0.37 & 0.64 & 0.27 \\
Yes & $13(3.6)$ & 8.13 & 1.68 & 0.31 & 0.70 & 0.28 \\
No & $341(95.0)$ & & & & & \\
Missing & $5(1.4)$ & & & & \\
\hline
\end{tabular}

a: For all analyses including PFHxS, $\mathrm{N}$ was 357 ,

*: $\mathrm{P}<0.05$ when comparing the PFAS concentrations in all categories of the characteristics, T-test for binary variables and F-test for variables with more than two categories

During the one-year period, the mean number of days for which the participants reported on the presence of symptoms was 314.2 (42-365). The mean number of days with any symptom was $65.7(0-305)$ and thus, the children on average experienced symptoms $23 \%$ of the time (65.7 days out of 314.2 days). A stuffed or runny nose was the most commonly reported symptom, appearing in 337 children and representing almost $16 \%$ of the time at risk, followed by cough (10\%), which was experienced by 335 children. At least one day with fever was reported by 283 children and seen on $1.6 \%$ of the days at risk during the one-year period. Diarrhea $(0.6 \%)$ and vomiting $(0.4 \%)$ were rare symptoms.

\begin{tabular}{|c|c|c|c|}
\hline $\begin{array}{l}\text { Chil } \\
\text { sym }\end{array}$ & $\begin{array}{l}\text { Aren experiencing } \\
\text { tom, } \mathbf{N}^{\mathrm{a}}\end{array}$ & $\begin{array}{l}\text { Mean number } \\
\text { of days (range) }\end{array}$ & $\begin{array}{c}\text { Mean proportion } \\
\text { of days }{ }^{\mathrm{b}} \text { (range) }\end{array}$ \\
\hline Any symptom & 354 & $65.7(0-305)$ & $23.0(0-100)$ \\
\hline Stuffed or runny nose & 337 & $46.3(0-291)$ & $15.7(0-83.1)$ \\
\hline Cough & 335 & $28.8(0-176)$ & $9.8(0-66.2)$ \\
\hline Felt unwell & 267 & $6.5(0-53))$ & $2.2(0-17.4)$ \\
\hline Temperature of 38.5 degrees or above & 283 & $4.7(0-35)$ & $1.6(0-10.3)$ \\
\hline Wheezy or whistling breathing & 98 & $2.1(0-48)$ & $1.1(0-100)$ \\
\hline Diarrhea & 143 & $1.6(0-29)$ & $0.6(0-14.8)$ \\
\hline Eye inflammation & 90 & $1.5(0-21)$ & $0.5(0-8.5)$ \\
\hline Ear pain & 108 & $1.5(0-49)$ & $0.5(0-18.4)$ \\
\hline Vomiting & 178 & $1.1(0-12)$ & $0.4(0-2.4)$ \\
\hline Discharge from ears & 37 & $0.7(0-43)$ & $0.3(0-25.6)$ \\
\hline Blood in stool & 4 & $<0.1(0-7)$ & $0.01(0-2.2)$ \\
\hline
\end{tabular}

\footnotetext{
${ }^{a}$ : The total number of participating children who experienced at least one day with the symptom during one year ${ }^{b}$ : The mean number of days experiencing a symptom divided by the mean number of days at risk of experiencing a symptom
} 
The adjusted results on the association between PFAS exposure and the days with symptoms as a binary variable below or above the median (model 1) are shown in Table 3 . The results were similar to the results found in the crude analysis (data not shown).

Higher serum concentrations of all PFASs tended to increase the odds of having a proportion of days with fever above the median, and this was statistically significant for PFOS and PFOA exposure in the high tertile as compared to the low tertile (IRR, PFOS: 2.35 (95\% CI: 1.34, 4.11), PFOA: 1.97 (95\%CI: 1.07, 3.62)). In contrast, higher exposure to PFNA and PFDA generally tended to reduce the odds of having days with symptoms above the median. This association was statistically significant for nasal discharge. No associations were statistically significant at the Bonferroni adjusted significance level. 


\begin{tabular}{|c|c|c|c|c|c|c|}
\hline & & Fever & Cough & Nasal discharge & Diarrhea & Vomiting \\
\hline PFOS & Low (0-6.93) & Reference & Reference & Reference & Reference & Reference \\
\hline \multirow[t]{2}{*}{$\mathrm{N}=346$} & Medium (6.94-10.18) & $1.41(0.81,2.44)$ & $1.16(0.67,2.01)$ & $1.11(0.65,1.93)$ & $0.89(0.51,1.56)$ & $1.47(0.86,2.54)$ \\
\hline & High (10.19-25.10) & $2.35 *(1.34,4.11)$ & $1.03(0.59,1.79)$ & $1.07(0.62,1.85)$ & $1.04(0.59,1.82)$ & $0.78(0.45,1.35)$ \\
\hline PFOA & Low $(0-1.27)$ & Reference & Reference & Reference & Reference & Reference \\
\hline \multirow[t]{2}{*}{$\mathrm{N}=346$} & Medium (1.28-2.03) & $1.55(0.90,2.95)$ & $0.72(0.42,1.24)$ & $1.19(0.70,2.04)$ & $1.10(0.64,1.89)$ & $1.05(0.62,1.78)$ \\
\hline & High (2.04-10.12) & $1.97 *(1.07,3.62)$ & $1.01(0.42,1.24)$ & $1.37(0.75,2.51)$ & $0.94(0.51,1.74)$ & $0.95(0.52,1.72)$ \\
\hline PFHxS & Low $(0-0.23)$ & Reference & Reference & Reference & Reference & Reference \\
\hline \multirow[t]{2}{*}{$\mathrm{N}=344$} & Medium (0.24-0.37) & $0.99(0.58,1.71)$ & $1.04(0.60,1.79)$ & $1.36(0.79,2.36)$ & $1.16(0.66,2.02)$ & $0.91(0.53,1.56)$ \\
\hline & High $(0.38-1.01)$ & $1.29(0.72,2.28)$ & $0.97(0.54,1.73)$ & $0.76(0.42,1.35)$ & $1.39(0.77,2.51)$ & $0.61(0.34,1.09)$ \\
\hline PFDA & Low $(0-0.22)$ & Reference & Reference & Reference & Reference & Reference \\
\hline \multirow[t]{2}{*}{$\mathrm{N}=346$} & Medium (0.23-0.31) & $1.07(0.63,1.81)$ & $0.63(0.37,1.07)$ & $0.59 *(0.35,1.00)$ & $0.91(0.53,1.56)$ & $0.90(0.53,1.51)$ \\
\hline & High (0.32-1.67) & $1.45(0.85,2.49)$ & $0.85(0.50,1.46)$ & $0.61(0.36,1.05)$ & $0.91(0.52,1.57)$ & $0.72(0.42,1.22)$ \\
\hline PFNA & Low $(0-0.55)$ & Reference & Reference & Reference & Reference & Reference \\
\hline \multirow[t]{2}{*}{$\mathrm{N}=346$} & Medium (0.56-0.81) & $1.00(0.59,1.71)$ & $0.74(0.43,1.27)$ & $0.53 *(0.31,0.92)$ & $0.75(0.44,1.30)$ & $0.89(0.53,1.52)$ \\
\hline & High (0.82-3.64) & $1.49(0.86,2.59)$ & $0.59(0.33,1.03)$ & $0.55^{*}(0.31,0.97)$ & $0.94(0.54,1.65)$ & $0.86(0.50,1.48)$ \\
\hline
\end{tabular}

Adjusted for maternal age, maternal educational level, parity and child age

${ }^{a}$ : Odds-ratio between low exposure, and medium and high exposure, respectively

*: $\mathrm{P}<0.05$ 
When the number of days with symptoms was used as the outcome (model 2), the crude and adjusted results were similar (data not shown) and the overall direction and size of the estimates were the same as when analyzing the outcome binary (model 1). However, the association between PFOS and fever was now statistically significant also after Bonferroni adjustment (Table 4). PFOS exposure in the high tertile as compared to the low tertile significantly increased the expected proportion of days with fever (IRR: 1.65 (95\% CI: 1.24; 2.18). There was a statistically significant linear trend across exposure groups $(\mathrm{p}<0.001)$, and the association was also present when PFOS was entered as a continuous variable; a $1 \mathrm{ng} / \mathrm{mL}$ increase in PFOS was associated to a $6 \%$ increase in the expected proportion of days with fever (IRR: 1.06 (95\% CI: 1.03; 1.09). The tendency of a reduced proportion of days with symptoms when exposed to higher levels of PFNA and PFDA was statistically significant at the 5\% significance level for diarrhea when comparing PFDA exposure in the medium to the low tertile (IRR: 0.46 (95 \% CI: 0.26, 0.81)). 


\begin{tabular}{|c|c|c|c|c|c|c|}
\hline & & Fever & Coughing & Nasal discharge & Diarrhea & Vomiting \\
\hline PFOS & Low $(0-6.93 \mathrm{ng} / \mathrm{mL})$ & Reference & Reference & Reference & Reference & Reference \\
\hline \multirow[t]{2}{*}{$\mathrm{N}=346$} & Medium (6.94-10.18 ng/mL) & $1.23(0.93,1.63)$ & $1.03(0.80,1.34)$ & $1.22(0.93,1.61)$ & $1.41(0.79,2.51)$ & $1.18(0.80,1.74)$ \\
\hline & $\operatorname{High}(10.19-25.10 \mathrm{ng} / \mathrm{mL})$ & $1.65 * *(1.24,2.18)$ & $0.88(0.67,1.15)$ & $1.02(0.76,1.35)$ & $1.19(0.67,2.12)$ & $0.87(0.58,1.31)$ \\
\hline PFOA & Low $(0-1.27 \mathrm{ng} / \mathrm{mL}))$ & Reference & Reference & Reference & Reference & Reference \\
\hline \multirow[t]{2}{*}{$\mathrm{N}=346$} & Medium $(1.28-2.03 \mathrm{ng} / \mathrm{mL})$ & $1.14(0.87,1.51)$ & $1.02(0.79,1.32)$ & $1.08(0.83,1.42)$ & $1.07(0.61,1.89)$ & $0.89(0.61,1.32)$ \\
\hline & High $(2.04-10.12 \mathrm{ng} / \mathrm{mL})$ & $1.12(0.82,1.54)$ & $1.00(0.75,1.33)$ & $1.04(0.76,1.41)$ & $1.08(0.55,2.13)$ & $0.95(0.62,1.47)$ \\
\hline PFHxS & Low $(0-0.23 \mathrm{ng} / \mathrm{mL})$ & Reference & Reference & Reference & Reference & Reference \\
\hline \multirow[t]{2}{*}{$\mathrm{N}=344$} & Medium $(0.24-0.37 \mathrm{ng} / \mathrm{mL})$ & $1.07(0.80,1.42)$ & $1.14(0.87,1.48)$ & $1.23(0.93,1.61)$ & $1.18(0.64,2.19)$ & $0.91(0.61,1.34)$ \\
\hline & $\operatorname{High}(0.38-1.01 \mathrm{ng} / \mathrm{mL})$ & $1.20(0.89,1.62)$ & $1.00(0.76,1.31)$ & $0.94(0.70,1.25)$ & $1.71(0.92,3.16)$ & $0.72(0.47,1.12)$ \\
\hline PFDA & Low $(0-0.22 \mathrm{ng} / \mathrm{mL})$ & Reference & Reference & Reference & Reference & Reference \\
\hline \multirow[t]{2}{*}{$\mathrm{N}=346$} & Medium $(0.23-0.31 \mathrm{ng} / \mathrm{mL})$ & $0.97(0.73,1.27)$ & $0.85(0.66,1.09)$ & $0.85(0.65,1.10)$ & $1.15(0.67,1.99)$ & $0.76(0.53,1.11)$ \\
\hline & $\operatorname{High}(0.32-1.67 \mathrm{ng} / \mathrm{mL})$ & $1.14(0.86,1.50)$ & $0.87(0.67,1.13)$ & $0.89(0.68,1.17)$ & $0.75(0.43,1.31)$ & $0.78(0.53,1.14)$ \\
\hline PFNA & Low $(0-0.55 \mathrm{ng} / \mathrm{mL})$ & Reference & Reference & Reference & Reference & Reference \\
\hline \multirow[t]{2}{*}{$\mathrm{N}=346$} & Medium $(0.56-0.81 \mathrm{ng} / \mathrm{mL})$ & $0.94(0.71,1.24)$ & $0.85(0.66,1.10)$ & $0.90(0.69,1.18)$ & $0.46 *(0.26,0.81)$ & $0.74(0.51,1.08)$ \\
\hline & $\operatorname{High}(0.82-3.64 \mathrm{ng} / \mathrm{mL})$ & $1.12(0.84,1.49)$ & $0.82(0.63,1.07)$ & $0.87(0.66,1.15)$ & $0.74(0.42,1.30)$ & $0.80(0.54,1.19)$ \\
\hline
\end{tabular}

Adjusted for maternal age, maternal educational level, parity and child age

${ }^{a}$ : Incidence-rate ratio comparing the medium or high exposure to low exposure

$*: \mathrm{P}<0.05$

**: $\mathrm{P}<0.002$ (Bonferroni adjusted) 
The results of the analyses in which the symptoms were combined are shown in table 5 (Model 3) and the tendencies were similar to those reported for the symptoms separately. Again, no notable differences were observed between the crude and adjusted analyses (data not shown). Greater exposures to PFOS, PFOA, and PFHxS tended to increase the expected proportion of episodes with fever and coughing. The same pattern was seen for PFOS and PFOA in regard to episodes with co-occurrence of fever and nasal discharge, and this tendency was statistically significant for PFOA exposure in the medium tertile as compared to the low tertile (IRR: 1.38 (95\%CI: $1.03,1.86)$ ). For PFNA and PFDA, no clear pattern was observed. 
Table 5: Adjusted associations (IRR ${ }^{\mathrm{a}}(95 \% \mathrm{CI})$ ) between maternal serum concentrations of PFAS and the number of episodes of co-occurrence of fever and coughing, and fever and nasal discharge among 346 children

\begin{tabular}{|c|c|c|c|}
\hline & & Fever and coughing & Fever and nasal discharge \\
\hline PFOS & Low $(0-6.93 \mathrm{ng} / \mathrm{mL})$ & Reference & Reference \\
\hline \multirow[t]{2}{*}{$\mathrm{N}=346$} & Medium (6.94-10.18 ng/mL) & $1.16(0.86,1.58)$ & $1.07(0.78,1.45)$ \\
\hline & High $(10.19-25.10 \mathrm{ng} / \mathrm{mL})$ & $1.33(0.99,1.80)$ & $1.28(0.94,1.73)$ \\
\hline PFOA & Low $(0-1.27$ ng/mL)) & Reference & Reference \\
\hline \multirow{2}{*}{$\mathrm{N}=346$} & Medium $(1.28-2.03 \mathrm{ng} / \mathrm{mL})$ & $1.22(0.91,1.64)$ & $1.38 *(1.03,1.86)$ \\
\hline & High (2.04-10.12 ng/mL) & $1.11(0.80,1.56)$ & $1.20(0.85,1.70)$ \\
\hline PFHxS & Low $(0-0.23 \mathrm{ng} / \mathrm{mL})$ & Reference & Reference \\
\hline \multirow[t]{2}{*}{$\mathrm{N}=344$} & Medium $(0.24-0.37 \mathrm{ng} / \mathrm{mL})$ & $1.14(0.84,1.54)$ & $0.97(0.72,1.31)$ \\
\hline & High $(0.38-1.01 \mathrm{ng} / \mathrm{mL})$ & $1.13(0.82,1.55)$ & $0.92(0.66,1.27)$ \\
\hline PFDA & Low $(0-0.22 \mathrm{ng} / \mathrm{mL})$ & Reference & Reference \\
\hline \multirow{2}{*}{$\mathrm{N}=346$} & Medium $(0.23-0.31 \mathrm{ng} / \mathrm{mL})$ & $0.91(0.68,1.23)$ & $0.89(0.66,1.20)$ \\
\hline & High $(0.32-1.67 \mathrm{ng} / \mathrm{mL})$ & $1.27(0.96,1.70)$ & $1.18(0.88,1.58)$ \\
\hline PFNA & Low $(0-0.55 \mathrm{ng} / \mathrm{mL})$ & Reference & Reference \\
\hline \multirow[t]{2}{*}{$\mathrm{N}=346$} & Medium $(0.56-0.81 \mathrm{ng} / \mathrm{mL})$ & $0.95(0.70,1.27)$ & $0.86(0.64,1.15)$ \\
\hline & High $(0.82-3.64$ ng/mL) & $1.02(0.76,1.38)$ & $0.90(0.66,1.22)$ \\
\hline
\end{tabular}

Adjusted for maternal age, maternal educational level, parity and child age

a: Incidence-rate ratio comparing the medium or high exposure to low exposure

*: $\mathrm{P}<0.05$ 
Repeating the adjusted negative binomial regression analyses (model 2 and 3) only for those who replied to at least 25 of 26 text messages $(\mathrm{N}=148)$ showed the same overall tendency. Further, adjusting for duration of breastfeeding, day-care attendance, maternal smoking and child sex did not materially change the estimates (data not shown).

\section{Discussion}

In this prospective study of 359 mother-child pairs, we found an association between higher maternal serum concentrations of PFOS and PFOA, and a higher prevalenc of fever during a one-year period among children aged 1-4 years. We observed a linear trend from the low to the high PFOS exposure group on the expected proportion of days with fever, suggesting a dose-response relationship, which was further supported by analyzing the exposure as a continuous variable. Additionally, PFOS and PFOA exposure tended to increase the number of episodes with co-occurrence of fever and cough or nasal discharge during the one-year period. Higher levels of PFDA and PFNA tended to decrease the prevalence of coughing, nasal discharge, vomiting and diarrhea, although a protective effect of PFAS exposure seems biologically implausible. Of the clinical outcomes investigated here, fever was assumed to be the most specific indicator of an affected immune system, and was also the symptom that showed the clearest association to PFAS exposure. Thus, fever seems to be an appropriate indicator to use in future studies.

Five previous birth cohort studies have been conducted examining the adverse effects of prenatal PFAS exposure on clinical infectious or allergic disease (Fei et al. 2010; Granum et al. 2013; Kishi et al. 2013; Okada et al. 2012; Wang et al. 2011). To our knowledge no previous studies have obtained information about fever as such but only about infectious diseases, as assessed by parental questionnaire or hospital diagnosis, and their findings appear to be somewhat inconsistent (Fei et al. 2010; Granum et al. 2013). In a Norwegian 
cohort study, a positive association between the maternal serum concentration of PFOA, PFNA and PFHxS and the number of episodes of common cold and gastroenteritis was found in 99 children up to the age of 3 years (Granum et al. 2013). We did not observe any clear associations between the exposures and the symptoms tied specifically to those diseases (cough, nasal discharge, diarrhea and vomiting) when analyzed separately. We did, however, observe a tendency of an increased prevalence of co-occurrence of both fever and cough, and fever and nasal discharge in children exposed to higher levels of PFOS and PFOA. This tendency was only statistically significant for PFOA in regard to the co-occurrence of fever and nasal discharge, even though PFOS, PFOA and PFNA concentrations in our study were higher than in the Norwegian study (median, PFOS: $5.5 \mathrm{ng} / \mathrm{ml}$; PFOA: $1.1 \mathrm{ng} / \mathrm{ml}$; PFNA: 0.3 $\mathrm{ng} / \mathrm{ml}$ ). Part of the difference in PFAS concentrations may be due to the fact that Granum et al. measured PFASs just before birth, whereas we measured in early pregnancy; serum-PFAS concentrations are known to decrease throughout pregnancy (Glynn et al. 2012). In addition, in the Norwegian study, fever was not assessed, and infections were not considered dependent on an elevated body temperature. Finally, the validity of our findings is supported by the fact that information on infections was collected prospectively every second week during one year, as compared to retrospectively once a year in the Norwegian study. Overall, the two studies suggest that prenatal exposure to PFASs may be immunotoxic.

In a Danish cohort study among 1,400 mother-child pairs, no association was found between prenatal exposure to PFOA and PFOS and incidence of hospitalization due to infectious diseases up to 11 years of age, even though PFOS and PFOA concentrations were higher than in the present study (mean PFOA: $5.6 \mathrm{ng} / \mathrm{ml}$ and PFOS: $35.3 \mathrm{ng} / \mathrm{ml}$ ) (Fei et al. 2010). However, hospitalizations only occur in severe cases of infections and may depend on sociodemographic circumstances, whereas we studied self-reported fever and four other symptoms associated with infections repeatedly during a one-year period. Also, prenatal 
exposures were previously found to affect vaccine responses at age 5 years, but not in older children (Grandjean et al. 2012) and hospitalization rates in children up to age 11 years may have been affected by unknown postnatal exposures as well as a variety of other factors. The findings in the present study suggest that PFASs may be able to affect the activation of the immune system, which seems biologically plausible. Briefly, fever arises due to temperature regulation in the hypothalamus after stimulation by endogenous pyrogens (cytokines) that are produced and released by macrophages or T-lymphocytes, when stimulated by bacteria or viruses. Animal studies have shown that both PFOS and PFOA exposure affects and possibly increases the release of fever inducing cytokines like IL-1, IL-6 and TNF- $\alpha$ after stimulation (DeWitt et al. 2011; Fair et al. 2011), which might explain why we observed an increase in the prevalence of fever in the highest exposure groups. This is supported by animal studies. In pregnant mice exposure to PFOS resulted in a decrease in natural killer cell activity, specific IgM response, and a decrease in lymphocyte subpopulations (Keil et al. 2008) in the offspring. Similar studies of mice have found a reduced spleen weight and affected PPARs after prenatal exposure to PFOA (Abbott et al. 2012; Hines et al. 2009). In addition, PFOS exposure has been shown to suppress immunity to influenza A in adult mice (Guruge et al. 2009).

A major strength of this study was the longitudinal design and the prospectively collected data. However, only $42 \%$ of the eligible pregnant women participated in the Odense Child Cohort and they were more likely to be non-smokers, older and nulliparous than nonparticipants (Kyhl et al. 2015). However, since the serum-PFAS concentrations as well as the health of the babies were unknown to the women at the time of inclusion, this factor is not likely to have affected our results. Further, it was possible to invite only 1,647 of the 2,547 active cohort members to participate in the study on infections, and only 649 of the mothers in the cohort had their serum-PFAS concentrations measured. However, the attrition is 
assumed to be random, and the participants with PFAS measurements did not differ from those without (Lind et al. 2016). Of the initially 439 eligible mother-child pairs for this sub study, almost $20 \%$ were excluded during the one-year study period due to lack of response, reducing the sample size to 359 and 346 in adjusted analyses. However, since the concentration of PFAS at this point was still unknown to the mothers and since the excluded mothers did not differ materially from those participating, we do not believe that the dropout introduced selection bias.

The maternal serum concentration of PFASs before GA 16 weeks was used as proxy for prenatal exposure since PFAS has been shown to cross the placenta (Gützkow et al. 2012; Needham et al. 2011). Serum concentrations of PFAS are known to decrease throughout pregnancy, but PFAS measurements at different time points during pregnancy are highly correlated (Glynn et al. 2012) and the compounds have long half-lives of 4-5 years (Olsen et al. 2007). The exposure level measured in early pregnancy was therefore believed to be representative of the exposure level during the entire pregnancy. Of the PFASs, PFOS clearly appeared to be most important and also appeared in the highest concentrations in maternal serum. To what extent other PFASs contribute to this tendency is difficult to determine due to the inter-correlations between the substances (range of correlation coefficients, 0.20-0.78). Data on infectious symptoms was self-reported, which possibly may cause misclassification. However, we have no reason to believe that it was differential since the women did not know their PFAS concentration when they were answering the text messages. Additionally, due to the frequent and repeated collection of data on symptoms, the risk of recall problems was minimized. Further, the parents were instructed to only register a rectal temperature above $38.5^{\circ} \mathrm{C}$. However, symptoms like nasal discharge and coughing could be due to allergic diseases rather than infection, but we did not obtain information about this. Incomplete response constituted a potential problem, as on average only $86 \%$ of the mothers answered 
the text message in each period and thereby reported information on symptoms for only 314 days during the one year study period. Non-response could be due to both the fact that the child did not have any symptoms or did have symptoms, which would either overestimate or underestimate the days with symptoms. Yet, the same tendencies of association between PFAS and fever were found among children with almost complete responses from mothers (at least 25 of 26 text message responses, $\mathrm{N}=148$ ) as for the entire study population, and thus bias seems unlikely.

We did not obtain information on childhood PFAS exposure. Prenatal exposure is associated with childhood exposure to PFASs as a major source of postnatal exposure is through breastfeeding. Further, previous studies have found the childhood serum-PFAS concentrations to be associated with reduced antibody concentrations after routine childhood vaccination at age 5 (Grandjean et al. 2012). Thus, it would be interesting to include PFAS levels in child serum in future studies. Additionally, we did not obtain information on treatment for fever or vaccinations during the study period, factors that clearly could have influenced the number of days with fever. Further, even though we tried to adjust for a wide range of potential confounders without notable changes in the results, unmeasured confounding is always of potential concern. Finally, a large number of analyses were performed and some of our results could perhaps represent a chance finding, although some tendencies remained significant even after Bonferroni adjustment.

\section{Conclusions}

We found a positive association between in utero exposure to PFOS and PFOA and the selfreported prevalence of days with fever in children aged 1-4 years, which may indicate an increased incidence of infections as a possible consequence of immunotoxic effects. We observed no clear associations between PFOS, PFOA, PFHxS, PFDA or PFNA and other 
common symptoms of infection when analyzed separately. However, episodes of cooccurrence of fever and coughing and fever and nasal discharge tended to increase with increasing concentrations of PFOS and PFOA. These findings are of public health interest as the children in our study on average experienced fever 5 days a year, which has not only personal but also financial impact (Yin et al. 2013). Future studies exploring the possible association between PFAS exposure and fever as a possible sensitive marker of childhood infections are needed. 


\section{References}

Abbott BD, Wood CR, Watkins AM, Tatum-Gibbs K, Das KP, Lau C. 2012. Effects of perfluorooctanoic acid (PFOA) on expression of peroxisome proliferator-activated receptors (PPAR) and nuclear receptor-regulated genes in fetal and postnatal CD-1 mouse tissues. Reprod. Toxicol. 33:491-505; doi:10.1016/j.reprotox.2011.11.005.

Bach CC, Henriksen TB, Bossi R, Bech BH, Fuglsang J, Olsen J, et al. 2015. Perfluoroalkyl Acid Concentrations in Blood Samples Subjected to Transportation and Processing Delay. PLoS One 10:e0137768; doi:10.1371/journal.pone.0137768.

Berger U, Kaiser MA, Kärrman A, Barber JL, Van Leeuwen SPJ. 2011. Recent developments in trace analysis of poly- and perfluoroalkyl substances. Anal. Bioanal. Chem. 400:16251635; doi:10.1007/s00216-011-4823-8.

Calafat A, Kuklenyik Z, Reidy JA, Caudill SP, Tully JS, Needham LL. 2007. Serum concentrations of 11 polyfluoroalkyl compounds in the US population: data from the National Health and Nutrition Examination Survey (NHANES) 1999-2000. Environ. Sci. Technol. 41: 2237-2242.

DeWitt JC, Peden-Adams MM, Keller JM, Germolec DR. 2011. Immunotoxicity of Perfluorinated Compounds: Recent Developments. Toxicol. Pathol. 40:300-311; doi:10.1177/0192623311428473.

Fair PA, Driscoll E, Mollenhauer MAM, Bradshaw SG, Yun SH, Kannan K, et al. 2011. Effects of environmentally-relevant levels of perfluorooctane sulfonate on clinical parameters and immunological functions in B6C3F1 mice. J. Immunotoxicol. 8:17-29; doi:10.3109/1547691X.2010.527868.

Fei C, McLaughlin JK, Lipworth L, Olsen J. 2010. Prenatal exposure to PFOA and PFOS and risk of hospitalization for infectious diseases in early childhood. Environ. Res. 110:7737; doi:10.1016/j.envres.2010.08.004.

Glynn A, Berger U, Bignert A, Ullah S, Aune M, Lignell S, et al. 2012. Perfluorinated alkyl acids in blood serum from primiparous women in Sweden. Env. Sci Technol 46:9071-9079; doi:10.1021/es301168c.

Grandjean P, Andersen EW, Budtz-Jørgensen E, Nielsen F, Mølbak K, Weihe P, et al. 2012. Serum vaccine antibody concentrations in children exposed to perfluorinated compounds. JAMA 307:391-7; doi:10.1001/jama.2011.2034.

Granum B, Haug LS, Namork E, Stølevik SB, Thomsen C, Aaberge IS, et al. 2013. Pre-natal exposure to perfluoroalkyl substances may be associated with altered vaccine antibody levels and immune-related health outcomes in early childhood. J. Immunotoxicol. 10:373-9; doi:10.3109/1547691X.2012.755580.

Guruge K, Hikono H, Shimada N, Murakami K, Hasegawa J, Yeung LWY, et al. 2009. Effect of perfluorooctane sulfonate (PFOS) on influenza A virus-induced mortality in female B6C3F1 mice. J. Toxicol. Sci. 34: 687-691.

Gützkow KB, Haug LS, Thomsen C, Sabaredzovic A, Becher G, Brunborg G. 2012. Placental transfer of perfluorinated compounds is selective--a Norwegian Mother and Child subcohort study. Int. J. Hyg. Environ. Health 215:216-9; doi:10.1016/j.ijheh.2011.08.011.

Hernán MA, Robins JM. 2015. Causal Inference.

Hines EP, White SS, Stanko JP, Gibbs-Flournoy EA, Lau C, Fenton SE. 2009. Phenotypic dichotomy following developmental exposure to perfluorooctanoic acid (PFOA) in 
female CD-1 mice: Low doses induce elevated serum leptin and insulin, and overweight in mid-life. Mol. Cell. Endocrinol. 304:97-105; doi:10.1016/j.mce.2009.02.021.

Jensen TK, Andersen LB, Kyhl HB, Nielsen F, Christesen HT, Grandjean P. 2015. Association between Perfluorinated Compound Exposure and Miscarriage in Danish Pregnant Women. P.B. Szecsied. PLoS One 10:e0123496; doi:10.1371/journal.pone.0123496.

Kato K, Wong L-Y, Basden BJ, Calafat AM. 2013. Effect of temperature and duration of storage on the stability of polyfluoroalkyl chemicals in human serum. Chemosphere 91:115-7; doi:10.1016/j.chemosphere.2012.11.013.

Kato K, Wong LY, Jia LT, Kuklenyik Z, Calafat AM. 2011. Trends in exposure to polyfluoroalkyl chemicals in the U.S. population: 1999-2008. Environ. Sci. Technol. 45:8037-8045; doi:10.1021/es1043613.

Keil DE, Mehlmann T, Butterworth L, Peden-Adams MM. 2008. Gestational exposure to perfluorooctane sulfonate suppresses immune function in B6C3F1 mice. Toxicol. Sci. 103:77-85; doi:10.1093/toxsci/kfn015.

Kishi R, Kobayashi S, Ikeno T, Araki A, Miyashita C, Itoh S, et al. 2013. Ten years of progress in the Hokkaido birth cohort study on environment and children's health: cohort profile--updated 2013. Environ. Health Prev. Med. 18:429-50; doi:10.1007/s12199013-0357-3.

Kyhl HB, Jensen TK, Barington T, Buhl S, Norberg LA, Jørgensen JS, et al. 2015. The Odense Child Cohort: aims, design, and cohort profile. Paediatr. Perinat. Epidemiol. 29:250-8; doi:10.1111/ppe.12183.

Lau C, Anitole K, Hodes C, Lai D, Pfahles-Hutchens A, Seed J. 2007. Perfluoroalkyl acids: a review of monitoring and toxicological findings. Toxicol. Sci. 99:366-94; doi:10.1093/toxsci/kfm128.

Lind DV, Priskorn L, Lassen TH, Nielsen F, Kyhl HB, Kristensen DM, et al. 2016. Prenatal exposure to perfluoroalkyl substances and anogenital distance at 3 months of age as marker of endocrine disruption. Reprod. Toxicol.; doi:10.1016/j.reprotox.2016.07.008.

Mogensen UB, Grandjean P, Nielsen F, Weihe P, Budtz-Jørgensen E. 2015. Breastfeeding as an Exposure Pathway for Perfluorinated Alkylates. Environ. Sci. Technol.; doi:10.1021/acs.est.5b02237.

Needham LL, Grandjean P, Heinzow B, Jørgensen PJ, Nielsen F, Patterson DG, et al. 2011. Partition of environmental chemicals between maternal and fetal blood and tissues. Environ. Sci. Technol. 45:1121-1126; doi:10.1021/es1019614.

Okada E, Sasaki S, Saijo Y, Washino N, Miyashita C, Kobayashi S, et al. 2012. Prenatal exposure to perfluorinated chemicals and relationship with allergies and infectious diseases in infants. Environ. Res. 112:118-25; doi:10.1016/j.envres.2011.10.003.

Olsen GW, Burris JM, Ehresman DJ, Froehlich JW, Seacat AM, Butenhoff JL, et al. 2007. Halflife of serum elimination of perfluorooctanesulfonate,perfluorohexanesulfonate, and perfluorooctanoate in retired fluorochemical production workers. Environ. Health Perspect. 115:1298-305; doi:10.1289/ehp.10009.

Romano ME, Xu Y, Calafat AM, Yolton K, Chen A, Webster GM, et al. 2016. Maternal serum perfluoroalkyl substances during pregnancy and duration of breastfeeding. Environ. Res. 149:239-246; doi:10.1016/j.envres.2016.04.034.

Sullivan JE, Farrar HC. 2011. Fever and antipyretic use in children. Pediatrics 127:580-7; 
doi:10.1542/peds.2010-3852.

Timmermann CA, Budtz-Jørgensen E, Petersen M, Weihe P, Steuerwald U, Nielsen F, et al. 2016. Shorter duration of breastfeeding at elevated exposures to perfluoroalkyl substances. Reprod. Toxicol.; doi:10.1016/j.reprotox.2016.07.010.

Vorkamp K, Nielsen F, Kyhl HB, Husby S, Nielsen LB, Barington T, et al. 2014. Polybrominated diphenyl ethers and perfluoroalkyl substances in serum of pregnant women: levels, correlations, and potential health implications. Arch. Environ. Contam. Toxicol. 67:9-20; doi:10.1007/s00244-013-9988-z.

Wang I-J, Hsieh W-S, Chen C-Y, Fletcher T, Lien G-W, Chiang H-L, et al. 2011. The effect of prenatal perfluorinated chemicals exposures on pediatric atopy. Environ. Res. 111:78591; doi:10.1016/j.envres.2011.04.006.

Yin JK, Salkeld G, Lambert SB, Dierig A, Heron L, Leask J, et al. 2013. Estimates and determinants of economic impacts from influenza-like illnesses caused by respiratory viruses in Australian children attending childcare: a cohort study. Influenza Other Respi. Viruses 7:1103-1112; doi:10.1111/irv.12138. 